\title{
Evolución, dos miradas desde la semántica de ciudadanía
}

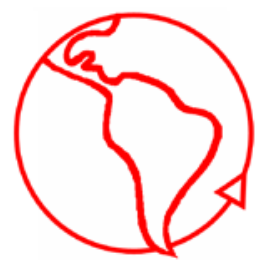

Evolution, two views from the semantics of citizenship

\section{Minda Bustamante Soldevilla}

Universidad Católica Santa María de Arequipa, Perú

\section{Karen Rosenfeld Ytier}

Universidad de Chile

Resumen

\begin{abstract}
La noción de evolución ha sido abordada desde diferentes perspectivas. Desde la Teoría de Sistemas Sociales, planteada por Luhmann, se plantea que ésta ocurre en los sistemas sociales, a partir de un proceso de tres selecciones. Utilizando esta perspectiva, puede entenderse cualquier cambio que a posteriori pueda atribuírsele un propósito o la idea de mejora y avance. En el presente artículo, se mostrará a través de la evolución del concepto ciudadanía cómo la sociedad moderna ha evolucionado dejando los rasgos adscritos para organizarse y definir otras formas de inclusión en los diversos sistemas parciales. Analizaremos aquí, cómo a través de la aplicación de la noción sistémico social de evolución se puede entender la forma en que se ha llegado a incluir en la semántica de ciudadanía a sectores tradicionalmente excluidos como son las mujeres y los miembros de pueblos indígenas. Es decir, la reestabilización del concepto inclusivo de ciudadanía, en un contexto de sociedad funcionalmente diferenciada.
\end{abstract}

\begin{abstract}
The notion of evolution has been approached from different perspectives. From the Theory of Social Systems, raised by Luhmann, we propose that this occurs in social systems, from a three selection process. Using this perspective, one can understand any change that later may be assigned a goal or the idea of improvement and advancement. This article will show through the evolving concept of citizenship as modern society has evolved leaving traits ascribed to organize and define other forms of inclusion in the various subsystems. We will discuss here how through the application of the concept of social system evolution can understand how that has come to include the semantics of citizenship traditionally excluded such as women and members of indigenous peoples. That is, re-stabilization of the inclusive concept of citizenship in the context of a functionally differentiated society.
\end{abstract}

Palabras Clave: Ciudadanía; evolución; ciudadanía indígena; ciudadanía mujer; inclusión/ exclusión

Keywords: Citizenship, evolution; indigenous citizenship; citizenship women; inclusion/ exclusion

\section{Introducción}

La noción de evolución ha sido abordada desde diferentes perspectivas en las cuales tiende a primar la idea de avance, mejora, progreso. Es decir, de las variaciones que ocurren tenderían a permanecer las que implicarían una mejor adaptación a un entorno, las que son más funcionales. Como si hubiera una intensión o propósito hacia un ideal 
óptimo, una direccionalidad previsible, por un lado, y una intervención directa desde el entorno. (Corsi, Esposito y Baraldi)

Desde la mirada sistémica social, en cambio, se plantea que todas éstas son atribuciones posteriores que realiza un observador, de un proceso de cambio ya ocurrido; el cual sucede como parte del operar de sistemas autorreferenciales autopoiéticos, determinados por su estructura y a partir de la incorporación de elementos irritantes provenientes de su entorno - Aquello que como sistema es capaz de captar e incluir dada su estructura, y hace parte de su operar sin que haya una ruptura del mismo, lo que puede entenderse como acoplamiento estructural.

Al mismo tiempo, no puede juzgarse o calificarse ni la adaptación ni los cambios evolutivos, pues un sistema existe mientras está adaptado, cuando ya no es posible la relación entre entorno y sistema sin que éste pierda su identidad, el sistema desaparece (se destruye o se constituye en otro sistema) (Corsi, Esposito y Baraldi)

En lo que sigue, se mostrará a través de la evolución del concepto ciudadanía cómo la sociedad moderna ha dejado de lado los rasgos adscritos a este concepto para organizarse y definir otras formas de inclusión en los diversos sistemas parciales. Analizaremos aquí, cómo a través de la aplicación de la noción de evolución de Luhmann se puede entender la forma en que se ha llegado a incluir en la semántica de ciudadanía a sectores tradicionalmente excluidos como son las mujeres y los integrantes de pueblos indígenas. Es decir, la reestabilización del concepto inclusivo de ciudadanía, en un contexto de sociedad funcionalmente diferenciada.

\section{Una mirada a los conceptos}

Desde esta perspectiva la evolución clásica, la modernización y el progreso son una constante dentro de cualquier sistema (de la vida, psíquico o social), determinado por su estructura y parte de sus operaciones. Por lo mismo ocurre como parte de la autopoiesis de los sistemas. En el caso de los sistemas sociales, la sociedad o un sistema parcial como la política, implica que en la concatenación de comunicaciones que los generan, la evolución (cambio en la estructura, es decir, en las expectativas que guían la comunicación) es algo que va a ocurrir pues las variaciones en las comunicaciones son una posibilidad (aunque cada comunicación en sí sea improbable)

Este proceso ocurre en tres fases, también concebidas como mecanismos, que a su vez se asocian a los componentes de la autopoiesis de los sistemas; estos mecanismos son variación, selección y reestabilización. Como se dijo, en los sistemas sociales esto ocurre en el plano de las comunicaciones en tanto éstas son sus elementos constituyentes, aún cuando Luhmann habla de adquisiciones evolutivas que también pueden implicar cambios físicos. Como toda la teoría sistémica, la evolución no tiene direccionalidad, no hay una normativa respecto hacia dónde debe ir esta evolución, más que la apertura de alternativas dada cada selección.

La variación, ligada a las comunicaciones (componente nivel elemento) ocurre como respuesta determinada estructuralmente a un cambio del entorno, es decir, son gatilladas en el sistema a partir de una perturbación en el acoplamiento sistema/ entorno. Siempre se requiere del entorno para evolución del sistema, aunque el primero no puede direccionar ésta. Las variaciones son distanciamientos, "reproducciones desviantes" (Dockendorff, 2006), de los elementos del sistema, los cuales pueden converger o divergir con, como dice Luhmann, lo aceptado en la sociedad, o, lo que Dockendorff plantea como, matriz cultural (como expectativas últimas transversales y no explicitadas). Si bien, siempre deben ser capaces de acoplarse a las comunicaciones anteriores como parte de la conservación de la autopoiesis del sistema, la semántica, subyacente o explícito de dicha comunicación puede ser coherente con la matriz cultural imperante (variación 
convergente) o plantear supuestos totalmente distintos, cuestionar la matriz imperante (variación divergente). En el caso de la ciudadanía, las comunicaciones del sistema político pueden continuar la reproducción del modo de entenderla o pueden plantear variaciones, o pueden surgir comunicaciones desde otros sistemas directamente relacionados con la ciudadanía o con algunos supuestos de base que impliquen algún cuestionamiento o variación.

La selección, puede a su vez escoger la variación, aceptándola, o rechazándola. En el caso de las variaciones convergentes, su aceptación implica conservar la matriz cultural o la estructura ya establecida, aunque puedan surgir leves modificaciones; en tanto, la aceptación de la variación divergente o el rechazo de la convergente puede llevar a un cambio en la estructura o expectativas, por ejemplo de quién es ciudadano.

En el caso de la ciudadanía, variación convergente puede ser el paso de las comunicaciones de la mirada ateniense a la romana, pues varían los "beneficios" que trae aparejados la ciudadanía manteniendo la no universalidad de la misma (sólo incluye a algunos).

Finalmente, la reestabilización implica que la selección realizada es incorporada a la estructura, manteniendo la unidad del contexto, y el operar del sistema. Esto significa que se acepta la distinción realizada, que el contenido de la comunicación pasa a formar parte de las expectativas y supuestos que orientan las comunicaciones y selecciones futuras. Cuando lo que se reestabiliza son las variaciones convergentes hay evolución manteniendo la matriz cultural (fortaleciéndola); mientras al reetabilizarse las divergentes puede llevar a un cambio en la matriz cultural, nueva ciudadanía.

\section{Deriva evolutiva de la ciudadanía. Inclusión de la mujer.}

Aunque mucho se habla de ciudadanía dando por sentada su comprensión, lo que éste concepto implica ha experimentado grandes variaciones hasta ser el concepto actual, propio de una sociedad funcionalmente diferenciada en que se eliminan los rasgos adscritos como forma de establecer inclusión o exclusión. En esta evolución es posible observar dos líneas, muy relacionadas, en que se observan las distinciones. Por un lado qué le corresponde a un ciudadano, qué implicancias tiene ser reconocido como ciudadano, y por otro, quién es considerado, o incluido, como ciudadano.

Hasta el momento este concepto se ha desarrollado ligado a un territorio, y a lo que ahora se conoce como Estado-nación, de modo que fenómenos como globalización, transnacionalización, Unión Europea pueden gatillar una nueva selección de la distinción.

Un ejemplo de la primera línea de distinciones es la comparación, como se mencionaba anteriormente, que se puede hacer entre la ciudadanía ateniense y la romana. La primera considera a aquel que gobierna y es gobernado, todos iguales en la toma de decisiones y en la obediencia en las leyes, pero ciudadanos eran sólo los que eran propietarios hombres de genealogía conocida, patriarcas, guerreros y propietarios- En el caso romano de ente político pasa a ente legal, ciudadano lo es a partir de propiedad y práctica de jurisprudencia, libre de actuar protegido por la ley, gozando de derechos e inmunidades (ejercer derechos propios más que toma de decisiones) (Peyron, 2002)

La segunda línea guarda relación con la incorporación de diferentes grupos o sectores haciendo cada vez más inclusivo o universal el concepto, ligado a las variaciones en la distinción de individuo.

En este apartado continuaremos desde la segunda de estas líneas, y específicamente en la inclusión de la mujer como ciudadana. Esta reestabilización se ligó tanto a las demandas feministas y las relaciones de género, como a comunicaciones relacionadas con otras 
distinciones (individuo, gobierno), con expresiones locales (territoriales). Aunque son procesos globales, observables en diferentes países, se dan particularidades relacionadas a los contextos, las otras comunicaciones observables en ellos. Esto resalta la contingencia da cada selección de comunicación, podrían ser otras y podrían ocurrir en otro momento

Debates o variaciones en las comunicaciones en torno a si la ciudadanía hace referencia a participación, derechos o suscripción a la ley, o si tiene que ver con la propiedad o capacidades, si su extensión implica igualdad o real autogobierno, una forma de manejar las demandas sociales (búsqueda de inclusión) o una toma de decisión efectiva, se han encontrado presentes en la evolución del concepto. Las selecciones de distinciones que respecto a ellas se han hecho se han acoplado o han facilitado las variaciones en el grupo que se considera ciudadano. Por lo tanto, puede atribuirse que como antesala a la extensión hacia la mujer debió ocurrir una extensión a todos los hombres, por un lado, y cambios en la concepción de gobierno por otro. Así, en un tiempo se relacionaba la ciudadanía con la virtud, y la preservación de la comunidad, de modo que el ciudadano no era quien participaba en la toma de decisiones sino que entregaba su vida a la patria, controlaba la Fortuna, la contingencia (Peyrou, 2002)

Al mismo tiempo, ocurrió un cambio en la concepción de individuo que fue incluyendo a diferentes sectores sociales, entre ellos a la mujer. Esta variación se fue reestabilizando en el sistema político principalmente, siendo en la mayoría de los países el inicio de nuevas variaciones; luego se observa la ampliación de derechos reconocidos hasta que se habla de plena igualdad y se explicita en las constituciones, considerando derechos de otros ámbitos (ya no solo el derecho a voto, si no al trabajo, autonomía decisional, sobre el propio cuerpo).

Contextualmente esto ocurre como parte del debate sobre el sufragio universal, por ejemplo en Francia surge como parte de la oposición a monarquía y la inclusión del pueblo; pero en cada país la práctica del ser ciudadano ha sido diversa. Ya sea por las semánticas de las comunicaciones o por el modo en que son seleccionadas (implantadas).

En esta visión temporal, la normativización de la ciudadanía, es decir, las comunicaciones oficiales sobre la misma, los cambios a la ley pueden ser tanto gatilladores como reestabilizaciones, dar pie a la variación o ser una selección de comunicaciones sobre el autogobierno.

Como se dijo, el sufragio universal se entrelaza con la emergencia del individuo y la noción de igualdad, lo que ocurre unido a demandas de sectores excluidos (el pueblo, hombres sin propiedad, sin educación) y reflexiones sobre determinados planteamientos ${ }^{1}$.

Desde la distinción de la soberanía del pueblo como resistencia a la tiranía, a otro en que prima la autonomía que considera al pueblo como un conjunto de individuos que se autogobiernan. El individuo-ciudadano reemplaza al ciudadano-propietario, se universaliza la ciudadanía a quienes se considera individuo, los excluidos del sufragio son los excluidos de la nación (aristócratas, extranjeros, criminales, marginados: niños, mujeres, enfermo mentales) (Peyrou, 2002), la mujer al ser considerada dependiente quedaban fuera.

Incluso cuando comienza a hablarse de Sufragio Universal (Francia 1848) continúa siendo para hombres: todo hombre mayor de 21 años sin restricción de capacidad o censo. A

\footnotetext{
${ }^{1}$ Se observa el sufragio universal como objetivo social, en la lucha por la integración y reconocimiento. En la reflexión sobre su validez como forma óptima de toma de decisiones, centrado en el autogobierno, rechazo a monarquía, etc. Y en la práctica electoral, una cosa es que se autorice la extensión del derecho a voto y otra que se ejerza sin cuestionamiento
} 
esto puede atribuírsele la intensión de encarar la concordia nacional, unidad social y fraternidad; pero no se pensaba en un acto de soberanía o un instrumento para generar el debate plural.

Si bien la democracia e igualdad política comienzan a aceptarse como comunicaciones, el sufragio universal era símbolo de república, paz social y estabilidad, pero la voluntad popular aún no era superior. Para esto se argumentaba la inmadurez del pueblo, y la necesidad de contar con sujetos autónomos y racionales para ejercer soberanía, para lo cual la educación es de extrema importancia y mientras ciertos sectores no contaran con estas herramientas, como las mujeres, no estaban preparados para el pleno ejercicio del derecho al sufragio. ${ }^{2}$

Mientras se mantuvieron las comunicaciones respecto a la mujer como de naturaleza inferior física y mental, y se la consideraba como parte del mundo privado e incluso dentro de éste, perteneciente al doméstico-familiar, no fueron observadas como individuos. La familia es una corporación, lo que las mantenía excluidas del sufragio y de la ciudadanía. Ya en el siglo XIX cuando estas concepciones comienzan a variar, al menos en las comunicaciones más públicas, se argumentaba que eran más manipulables y que estaban más influenciadas por la Iglesia (lo que chocaba con la intensión de separar lo político de la Iglesia) En otros casos, (EE.UU. y Gran Bretaña) en que imperaba una visión más utilitarista estas mismas consideraciones constituyeron a las mujeres en un grupo aparte, con especificidades e intereses particulares, los cuáles era necesario recoger. La postura francesa en cambio parte de una contradicción entre universalismo de los derechos políticos individuales y el universalismo de las diferencias sexuales en que las diferencias de sexo son naturales y no trascendibles, lo que pone a las mujeres en desventaja, limitada a la función reproductora.

Esta desventaja se refuerza por la reivindicación hecha por el feminismo en ese entonces, que resaltaba las diferencias para ser tomadas en cuenta, no ser homologadas a los hombres, y en ese sentido ser iguales, pero a la vez ser distintas. Esto cuestionaba lo abstracto y neutro del individuo detentador de derechos, personas iguales y libres (sin distinción) francés. Además, de las investigaciones científicas que confirmaban la diferencia (e inferioridad) cognitiva y biológica (reproductiva) de la mujer.

Estas distinciones perduraron hasta la industrialización en que la mujer se incorporó al mundo laboral ya no sólo reproduciendo los trabajos realizados dentro del hogar. La transformación del arquetipo de mujer ha sido una evolución larga que, en cuanto evolución, continúa.

En lo que se atribuye como lucha de las mujeres por eliminar la exclusión del ámbito político, y lograr pleno ejercicio de derechos y ciudadanía entonces, las comunicaciones han girado entorno tanto a la igualdad como a la diferencia de género. Pero no puede sostenerse que se trata sólo de mejorar la argumentación para que estas comunicaciones sean seleccionadas, se requiere variación también en otras comunicaciones, de otros y nuevos actores, el devenir contextual. Las comunicaciones desde las mujeres pueden considerarse entorno a la esfera política hasta que no logran el reconocimiento al derecho a manifestarse.

Desde el presente puede describirse que las mujeres siguieron diversos caminos para lograr la ciudadanía plena, pero también tuvo relación con los cambios políticos, guerras o conflictos que surgían en cada territorio. Por esto, para lograr la reestabilización un cambio en las estructuras del sistema, las comunicaciones relacionadas deben ser

\footnotetext{
${ }^{2}$ Al menos en Francia se dio una extensión paulatina, en 1930 se incluye a los criados, 1944 a las mujeres y en 1975 los indigentes
} 
masivas. La comprensión no siempre es posible, de modo que para lograr la aceptación y selección es necesario que se compartan ya ciertas expectativas o haya un punto de encuentro en las comunicaciones previas.

Luego del logro de la inclusión en el derecho a sufragio, las demandas se extienden con mayor fuerza a otros ámbitos. La idea de ciudadanía amplía el campo de acción y las exigencias de igualdad de derechos. Se ha sumado a los cambios de la sociedad moderna abriéndose a manifestaciones mundiales, incorporándose en organismos internacionales, haciendo aún más notorias las posturas divergentes entre comunicaciones feministas: igualdad de derechos con los hombres versus afirmar la diferencia y la especificidad de las mujeres (Nash, 2005)

No obstante la aparente extensión de las comunicaciones feministas y sus argumentos, las reflexiones surgidas en ellas, las demandas y argumentaciones, son observables a dos niveles: elites y bases. Las segundas se movían en torno a preocupaciones concretas como la desvinculación de la sexualidad de la maternidad, campañas para legalizar anticonceptivos y a favor del aborto, denuncia pública de la violencia de género, lucha contra la criminalización del lesbianismo (Nash 2005). Todas ellas y otras, actualmente con cabida y espacio de manifestación gracias su reconocimiento como ciudadanas.

Por último, en esta evolución si bien se observa un aumento de los derechos otorgados y una mayor selección de las variaciones que refuerzan la presencia y reconocimiento de las mujeres aún falta reestabilización de algunas de ellas.

\section{Lo que ocurre en Chile.}

Las demandas de las mujeres en Chile comenzaron por los derechos civiles, las prácticas de libertad individual, de modo más notorio a mediados del siglo XIX, siendo el tema del sufragio poco relevante, incluso rechazado. Sin embargo, se dieron cuenta (desde un atribución a posteriori) que la participación política era esencial para lograr otros derechos, de modo que el derecho a voto se transforma en una exigencia (Errázuriz, 2005) Para que estas comunicaciones divergentes fueran seleccionadas fue necesario que las mujeres ocuparan los espacios públicos.

Las primeras manifestaciones públicas de las mujeres fueron a través del periódico "Eco de las Señoras de Santiago" en que se hacía una defensa a la Iglesia Católica, lo que se unió al tema de la "Cuestión Social" y la dedicación de las mujeres de clase alta a la beneficencia y caridad. Estos ámbitos comunicativos permitieron a su vez el surgimiento de comunicaciones sobre las mujeres en que se abogaba por una mejor condición de vida. En tanto la incorporación de las mujeres al mundo laboral, a partir de la necesidad generada por la crisis económica mundial, también promovió nuevas comunicaciones sobre los derechos de las mujeres.

Unas de las primeras selecciones de las variaciones son la inscripción de las mujeres en registros electorales (1875) y la aceptación de las mismas en la Universidad (1877). A esto siguió una progresiva organización y visibilización pública, pero que de todos modos fue lenta en un principio y alejada de la demanda por el derecho a voto; más interesada en el derecho a la educación e igualdad ante la ley - lo que se observa en la lenta inscripción en los registros electorales y baja participación en las elecciones, es decir, las comunicaciones divergentes no pueden homologarse a todo un sector- .

En 1934 se acepta el sufragio municipal, justificándolo desde las comunicaciones masculinas como modo de prueba para evaluar el comportamiento electoral e influencia en el equilibrio partidista. La aceptación del derecho a voto presidencial de la mujer o sufragio amplio (1949), en tanto, se dio en el contexto de declaración de ilegalidad del Partido Comunista (1948), amenaza a la imagen democrática de Chile, luego de que el 
proyecto elaborado por dos mujeres de organización Memch estuviera desde 1941 en el Congreso (Errazúriz, 2005). Obtienen así una ciudadanía activa (en la concepción nacional) pues pueden participar del sistema político en la expresión de sus exigencias. Sin embargo, un contexto favorable a la selección de alguna de las variaciones de las comunicaciones no implica necesariamente un cambio profundo en la concepción sobre las mujeres (aceptación de la divergencia), como se observa tienen gran peso las otras comunicaciones del ámbito político. A la vez que las comunicaciones a nivel de sistema parcial legal, por ejemplo, no implica grandes cambios en lo cotidiano en otros sistemas parciales económicos, familiar, educacional, organizacionales o interaccionales. Sin desmedro de lo cual, es una primera selección que abre la posibilidad de probabilizar las comunicaciones en torno a la igualdad de las mujeres en su condición de individuo sujeto de derechos y deberes.

Otras reestabilizaciones pueden observarse en la creación del Servicio de la Mujer, cambios a la Constitución, las Cuotas de representatividad, encuentros internacionales (Beijing), la ONU mujer y el posible Ministerio de la Mujer.

\section{Deriva evolutiva del concepto de ciudadanía: ciudadanías indígenas}

Con la evolución de la sociedad y su complejización se va también ampliando y complejizando el concepto de ciudadanía. La definición de ciudadanía y lo que entendemos por ella ha estado ligado a las ideas de sociedad que se tenían en cada uno de los momentos en que la sociedad iba cambiando desde una sociedad segmentada hasta una funcionalmente diferenciada.

Siendo característico de cada tipo de sociedad solamente la inclusión de algunos en la toma de decisiones políticas, en el manejo y gobierno de los Estados. Generalmente las normas definían quienes podían ejercer el derecho de ciudadanía, estado desde un principio destinada solamente a hombres letrados, con poder económico, conforme la evolución de las sociedades se venia dando. La inclusión de los excluidos fue parte del proceso evolutivo también, hasta llegar al momento actual en el que el concepto es completamente incluyente y la ciudadanía puede ser ejercida por todos y todas. Como hemos visto, primero se otorgo el derecho a voto a las mujeres, quienes después de luchas y movimientos sociales lograron constituirse como ciudadanas a cabalidad.

En el caso de los pueblos indígenas la deriva del proceso evolutivo fue diferente ya que el derecho y la inclusión se dieron desde reformas básicamente legales, desde las normas y desde las Constituciones, en diferentes procesos que han ido desde la integración hasta la ciudadanía diferenciada producto de Convenios internacionales y reivindicaciones legales hechas por los integrantes de pueblos indígenas.

En el caso de América Latina, con una alta densidad de poblaciones indígenas el cambio tenia que ser también evidente, las diferentes constituciones latinoamericanas han ido incorporando en sus cuerpos legales este concepto e incluyendo dentro de los derechos los derechos ciudadanos que les corresponden a los pueblos indígenas, el derecho a ser iguales ante la ley pero con diferencias, diferencias también en la implementación de políticas publicas desde el estado que les beneficien en su diferenciación étnica.

En el presente artículo nos proponemos mostrar como es que el concepto de ciudadanía y la amplitud de derechos que este abarca, ha ido avanzando a raíz de la suscripción por parte de los Estados Latinoamericanos Convenios internacionales y declaraciones de las naciones Unidas que han generado inclusión y una nueva corriente de ciudadanías diferenciadas. 


\section{Formas de gobierno en América Latina y ciudadanía indígena.}

Sin retroceder mucho en el tiempo, trataremos de centrarnos en las formas de Gobierno que hoy tienen los Estados de América Latina, Estados de tradición Republicana y en las últimas décadas con formas de gobierno democráticas.

La importancia de las cartas constitucionales radica que en ella generalmente se condensan los derechos fundamentales de los integrantes de un país, los principios que regirán la vida de las sociedades y sus integrantes de manera territorial. Citando a Willke (2006) podemos decir lo siguiente: Contrariamente a la posición defendida por Rudolf Stichweh (1995) en apoyo a Luhmann, entiendo la sociedad moderna no precisamente como una sociedad mundial, sino, tanto ahora como antes, bajo la forma de una unidad normativa territorialmente delimitada de (auto)organización social. Sólo unidades normativas territoriales están en posición de generar autónomamente las estructuras fundantes de su autoorientación. La autonomía es, en este sentido, una condición imprescindible para la formación de sistemas sociales autorreproductivos.

Siguiendo a Willke en esta concepción, la de que los estados son una unidad normativa territorialmente delimitada, analizaremos como es que esbozan en su carta magna la forma de organización del gobierno y de organización territorial:

\begin{tabular}{|c|c|c|c|c|c|}
\hline & $\begin{array}{l}\text { Argentina } \\
\text { (1853) }\end{array}$ & Chile (1980) & Colombia (1991) & Bolivia (2009) & Perú (1993) \\
\hline $\begin{array}{l}\text { Formas } \\
\text { de } \\
\text { Estado }\end{array}$ & $\begin{array}{l}\text { Articulo 10.- } \\
\text { La nación } \\
\text { Argentina } \\
\text { adopta para } \\
\text { su gobierno la } \\
\text { forma } \\
\text { representativa } \\
\text { Republicana } \\
\text { federal, según } \\
\text { lo establece la } \\
\text { presente } \\
\text { constitución. }\end{array}$ & $\begin{array}{l}\text { 3o.- El Estado de } \\
\text { Chile es unitario. } \\
\text { La } \\
\text { administración } \\
\text { del } \\
\text { Estado será } \\
\text { funcional } \\
\text { y territorialmente } \\
\text { descentralizada, } \\
\text { o } \\
\text { desconcentrada } \\
\text { en su } \\
\text { caso, } \\
\text { conformidad de } \\
\text { a la ley. } \\
\text { Artículo } \\
\text { Chile es una } \\
\text { república } \\
\text { democrática. }\end{array}$ & $\begin{array}{l}\text { Articulo 10.- } \\
\text { Colombia es un } \\
\text { Estado social de } \\
\text { derecho, } \\
\text { organizado en } \\
\text { forma de } \\
\text { República } \\
\text { unitaria, } \\
\text { descentralizada, } \\
\text { con autonomía } \\
\text { de sus entidades } \\
\text { territoriales, } \\
\text { democrática, } \\
\text { participativa } \\
\text { pluralista, } \\
\text { fundada en el } \\
\text { respeto de la } \\
\text { dignidad en el } \\
\text { humana, en en la } \\
\text { trabajo y la } \\
\text { solidaridad de las } \\
\text { personas que la } \\
\text { integran y en la } \\
\text { prevalencia del } \\
\text { interés general }\end{array}$ & 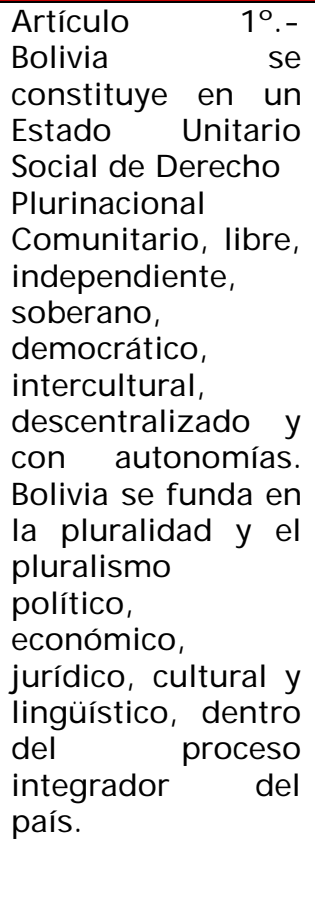 & 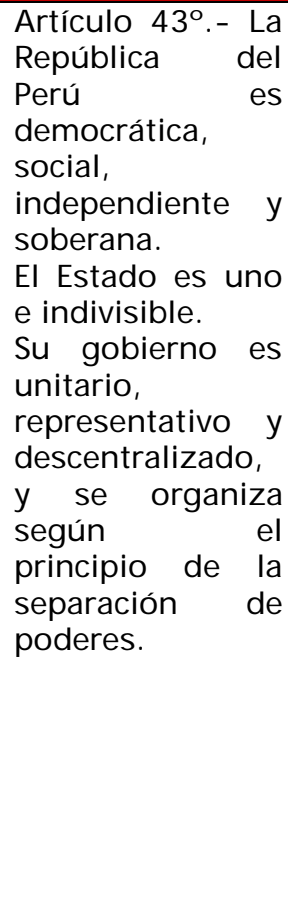 \\
\hline
\end{tabular}

Fuente: Elaboración Propia

En cada una de las constituciones analizadas, podemos ver que en general cada uno de los estados sigue adoptando la forma de organización Republicana, forma de organización que viene desde la fundación de los Estados nación después de la Independencia de España.

No detallaremos cada una de las constituciones ya que lo que nos interesa en este artículo es ver cómo es que ha evolucionado la deriva del concepto de ciudadanía en el caso de las 
ciudadanías indígenas, considerando que todos los países analizados tienen población indígena en mayor o menor medida.

Casi todas las constituciones reconocen como forma organizativa la republicana, menos en el caso de Bolivia (su constitución data de 2009) cuya organización es un Estado plurinacional, descentralizado y con autonomías.

\begin{tabular}{|c|c|c|c|c|c|}
\hline & $\begin{array}{l}\text { Argentina } \\
(1853)\end{array}$ & Chile (1980) & $\begin{array}{l}\text { Colombia } \\
\text { (1991) }\end{array}$ & Bolivia (2009) & Perú (1993) \\
\hline Ciudadanía & $\begin{array}{l}\text { Artículo } 8^{\circ} .- \\
\text { Los } \\
\text { ciudadanos } \\
\text { de cada } \\
\text { provincia } \\
\text { gozan de } \\
\text { todos los } \\
\text { derechos, } \\
\text { privilegios e } \\
\text { inmunidades } \\
\text { inherentes al } \\
\text { título de } \\
\text { ciudadano en } \\
\text { las demás } \\
\text { provincias. }\end{array}$ & $\begin{array}{l}\text { Artículo 13o.- } \\
\text { Son } \\
\text { ciudadanos los } \\
\text { chilenos } \\
\text { que hayan } \\
\text { cumplido } \\
\text { dieciocho años } \\
\text { de edad } \\
\text { y que no hayan } \\
\text { sido } \\
\text { condenados a } \\
\text { pena aflictiva. } \\
\text { La calidad de } \\
\text { ciudadano } \\
\text { otorga } \\
\text { los derechos de } \\
\text { sufragio, de } \\
\text { optar } \\
\text { a cargos de } \\
\text { elección } \\
\text { popular y los } \\
\text { demás } \\
\text { que la } \\
\text { Constitución } \\
\text { o la ley } \\
\text { confieran. }\end{array}$ & $\begin{array}{l}\text { Artículo 989-- } \\
\text { Mientras la ley } \\
\text { no decida otra } \\
\text { edad, la } \\
\text { ciudadanía se } \\
\text { ejercerá a partir } \\
\text { de los } \\
\text { dieciocho años. } \\
\\
\text { Articulo 99. La } \\
\text { calidad de } \\
\text { ciudadano en } \\
\text { ejercicio es } \\
\text { condición } \\
\text { previa e } \\
\text { indispensable } \\
\text { para ejercer el } \\
\text { derecho de } \\
\text { sufragio, para } \\
\text { ser elegido y } \\
\text { para } \\
\text { desempeñar } \\
\text { cargos públicos } \\
\text { que lleven } \\
\text { anexa } \\
\text { autoridad o } \\
\text { jurisdicción }\end{array}$ & $\begin{array}{l}\text { Articulo 1440. I } \\
\text { Son ciudadanas y } \\
\text { ciudadanos todas } \\
\text { las bolivianas y } \\
\text { todos los } \\
\text { bolivianos, y } \\
\text { ejercerán su } \\
\text { ciudadanía a } \\
\text { partir de los } 18 \\
\text { años de edad, } \\
\text { cualesquiera } \\
\text { sean sus niveles } \\
\text { de instrucción, } \\
\text { ocupación o } \\
\text { renta. }\end{array}$ & $\begin{array}{l}\text { Artículo } 30^{\circ} .- \\
\text { Son ciudadanos } \\
\text { los peruanos } \\
\text { mayores de } \\
\text { dieciocho años. } \\
\text { Para el ejercicio } \\
\text { de la ciudadanía } \\
\text { se requiere la } \\
\text { inscripción } \\
\text { electoral. }\end{array}$ \\
\hline
\end{tabular}

Fuente: Elaboración Propia

En el caso de la definición de ciudadanías podemos ver claramente el cambio en las definiciones, generalmente la ciudadanía se identifica con la nacionalidad eso lo podemos ver explícitamente en las Constituciones de Chile, Perú y Bolivia, en el caso de Argentina y Colombia no es explicita pero si puede inferirse.

La ciudadanía puede ser ejercida en todos los casos por los nacionales mayores de 18 años y desde ese pueden ejercer los derechos que reconocen las constituciones y las leyes.

En Perú la ciudadanía va aparejada con el derecho de sufragio que es obligatorio, identificando se en estos casos el derecho a voto con el derecho de ciudadanía. 
Bolivia describe en su carta magna una ciudadanía inclusiva desde el lenguaje, distinguiendo entre Bolivianos y Bolivianas.

En el caso de Chile y Colombia la calidad de ciudadano es requisito previo para poder ejercer el derecho de sufragar elegir y ser elegido.

La deriva evolutiva del concepto de ciudadanía ha pasado por los tres pasos del proceso de evolución (selección, variación y reestabilización) la reestabilización en este caso es legal y constitucional, pero no siempre real, por lo tanto aquí podemos distinguir entre la reestabilización legal y la real. Si bien en todas las constituciones, basta con ser mayor de 18 años y nacional de alguno de los países para ejercer los derechos que reconoce la ley, a este fenómeno podemos llamar ciudadanía legal en cambio en el caso de los países con presencia de pueblos indígenas el ejercicio de derechos no es igualitaria, por el contrario en muchas ocasiones se les ha considerado como ciudadanos de segunda clase. En el año 2009 en el Perú se da el conflicto de Bagua en el que los indígenas peruanos reclamaban el respeto de sus tierras ancestrales y el Presidente Alan García responde indicando lo siguiente: "ya está bueno, estas personas no tienen corona, no son ciudadanos de primera clase que puedan decirnos 400 mil nativos a 28 millones de peruanos..."(peru.com, 2009) podemos ver de esta forma que los indígenas no ejercen una ciudadanía real y esta es más bien solamente legal.

\section{Instrumentos de reducción de complejidad que aumentan la complejidad.}

Como hemos visto, el concepto de ciudadanía hoy es omniabarcador e igualitario, basta con ser nacional de un país y ser mayor de edad para serlo. Los indígenas también son ciudadanos de cada uno de los países de los que forman parte incluso antes de que se organicen como repúblicas. Dos instrumentos han modificado de alguna manera la deriva evolutiva de las ciudadanías indígenas, otorgando a los pueblos originarios y por ende a sus integrantes derechos colectivos que los diferencian del resto de ciudadanos de cada uno de las Repúblicas Latinoamericanas.

Podemos decir así que en la actualidad existen dos instrumentos internacionales que han sido ratificados y suscritos por los Estados que reducen la complejidad al establecer los derechos de manera escrita pero que la aumentan ya que para la efectiva aplicación de ellos se requiere concertaciones, nuevas legislaciones e investigaciones de cómo es que se podrá lograr el ejercicio cabal de esas nuevas formas de ciudadanía diferenciada.

La ciudadanía no es una condición (a)histórica: depende de los contextos. Hay contextos que son un obstáculo para el ejercicio de la ciudadanía, a pesar de que la ley la reconoce a todos por igual. Es el caso de la pobreza: en ella, como decíamos, se juntan la exclusión socioeconómica - la carencia de recursos - y la exclusión cultural, pues las culturas subalternas son culturas excluidas: culturas sin funciones públicas. La pobreza - decíamos por ello - es exclusión de la ciudadanía. No se soluciona con el otorgamiento de derechos especiales. Las leyes no traen como consecuencia inmediata la actuación de los derechos (Tubino 2008).

Podemos ver de esta manera que ser o no ser ciudadano depende de la legalidad plasmada en las Constituciones pero que sin embargo ejercer la ciudadanía depende de otros factores, por lo tanto la reestabilización del concepto de ciudadanía es legal y es real, pero en el caso de los indígenas es por el momento casi solamente legal. 


\begin{tabular}{|c|c|c|c|c|c|}
\hline & $\begin{array}{l}\text { Argentina } \\
\text { (1853) }\end{array}$ & Chile (1980) & Colombia (1991) & Bolivia (2009) & Perú (1993) \\
\hline $\begin{array}{l}\text { Convenio } \\
169 \text { OIT }\end{array}$ & Julio 2000 & Marzo 2009 & Agosto de 1991 & Diciembre 1991 & Febrero de 1994 \\
\hline $\begin{array}{l}\text { Declaración } \\
\text { ONU }\end{array}$ & La Asa & ea General de & ONU adopta la De & Iaración en Septie & abre de 2007 \\
\hline $\begin{array}{l}\text { Diferencias } \\
\text { culturales } \\
\text { reconocidas } \\
\text { en la } \\
\text { Constitución }\end{array}$ & No & No & $\begin{array}{l}\text { Artículo } 7^{\mathbf{0}} \text { - } \\
\text { El Estado } \\
\text { reconoce y } \\
\text { protege la } \\
\text { diversidad étnica } \\
\text { y cultural de la } \\
\text { Nación } \\
\text { colombiana. }\end{array}$ & $\begin{array}{l}\text { Artículo 30. Il. En } \\
\text { el marco de la } \\
\text { unidad del Estado } \\
\text { y de acuerdo con } \\
\text { esta Constitución } \\
\text { las naciones y } \\
\text { pueblos } \\
\text { indígenas } \\
\text { originarios } \\
\text { campesinos } \\
\text { gozan de los } \\
\text { siguientes } \\
\text { derechos:....) }\end{array}$ & $\begin{array}{l}\text { Articulo 2. Todo } \\
\text { peruano tiene } \\
\text { derecho: } \\
\text { 19.- A su } \\
\text { identidad étnica } \\
\text { y cultural. El } \\
\text { Estado reconoce } \\
\text { y protege la } \\
\text { pluralidad étnica } \\
\text { y cultural de la } \\
\text { nación. }\end{array}$ \\
\hline
\end{tabular}

Fuente: Elaboración propia

\section{Convenio 169 OIT y declaración de las Naciones Unidas sobre derechos de los pueblos indígenas.}

La ratificación del convenio, así como la suscripción por parte de los Estados miembro de las Naciones Unidas de la declaración de los Derechos de los Pueblos Indígenas, ha generado cambios legales y constitucionales desde que fueron reconocidos por los Estados. En el caso de Colombia, Perú y Bolivia, el Convenio fue Ratificado en la década de los 90 y las modificaciones Constitucionales vinieron casi en paralelo con dicha ratificación. Desde ese momento los tres estados reconocen diferencias culturales al interior de las Repúblicas. LA suscripción de la Declaración de los Derechos Indígenas será en el caso de Colombia y Perú simplemente complementaria a la ratificación del Convenio y cambios constitucionales. Bolivia, cambia de Constitución en 2009 después de haber suscrito la Declaración incorporando en su texto constitucional los derechos que prescribe el Convenio así como lo establecido por la Declaración.

En el caso de Argentina y Chile la ratificación del Convenio es reciente, y no ha habido cambios constitucionales, podríamos especular sosteniendo que una vez suscrita la Declaración de los Derechos de Los pueblos indígenas existió de alguna forma una presión política para la ratificación del Convenio, para ello en el caso de Chile “... Aun cuando el derecho a la libre determinación de los pueblos, está inscrito en el Derecho internacional desde la carta de las Naciones Unidas de 1945, una ultima declaración de la ONU ha intentado desparadojizar lo que se supone era paradoja indicando que los pueblos indígenas tienen derecho a la libre determinación..." (Mascareño 2010)

Aun quedan desafíos en el caso de todos los países de la Región para que la ciudadanía pueda ser ejercida da cabalidad por los integrantes de pueblos indígenas reconociendo las diferencias que para ellos conllevaría la evolución del concepto de "ciudadanía" al reconocerse sus particularidades étnicas y culturales. En el caso de Chile y Argentina aun queda por investigar y por hacer, estaremos atentos. 


\section{Conclusiones}

Después de haber analizado la evolución del concepto de ciudadanía con dos derivas diferentes podemos concluir lo siguiente:

1. La evolución de la "ciudadanía" en ambos casos tiene dos derivas diferentes que han confluido en algunos momentos. En el caso de las mujeres se inician movimientos y reivindicaciones propias que culminan en un reconocimiento legal. En el caso de los indígenas el reconocimiento legal es primero, producto del trabajo de intelectuales y académicos indígenas y no indígenas, la lucha por el ejercicio a cabalidad de las ciudadanías es una lucha constante.

2. En ambos casos (mujeres e indígenas) hemos observado muchas variaciones, menos selecciones y pocas reestabilizaciones.

3. en el caso de la mujeres ellas estaban más incluidas dentro de la sociedad, en el caso de los indígenas la integración al estado nación los invisibiliza y es recién hace pocas décadas que se viene reclamando derechos culturales y ciudadanías diferenciadas. RM

\section{Bibliografía}

Corsi, G., Espósito, E. \& Baraldi, C. (1996). Glosario sobre la Teoría Social de Niklas Luhmann. Barcelona: Antrophos.

Dockendorff, C. (2006). Evolución de la cultura: la deriva semántica del cambio estructural. Persona y Sociedad, XX(1), 45-73.

Errázuriz, J. (2005). Discursos en torno al sufragio femenino en Chile 1865-1949. Historia, II(38), 257- 286.

Nash, M. (2004). Mujeres en el mundo. Historia, retos y movimientos (reseña). Barcelona: Alianza editorial

Mascareño, A. (2010) Algunas veces subir es bajar. Paradojas de los derechos culturales. En O. Ette y H. Nitschack (Eds.),Trans*Chile. Un acercamiento transreal. Frankfurt a. M.: Iberoamericana- Vervuert Verlag.

Peyron, F. (2002). Perspectivas Historiográficas. Ciudadanía e Historia en Torno a la Ciudadanía. Revista Historia Social, (42), 145- 166.

Tubino, F. (2008). No una sino muchas ciudadanías una reflexión desde el Perú y América Latina. Cuadernos Interculturales Universidad de Valparaiso, 170- 180.

Willke, H. (2006). La transformación de la democracia como modelo de orientación de las sociedades complejas. Estudios Publicos, (102), 179- 201.

Fuentes electrónicas:

BCN (1980) Constitución Política de Chile http://www.bcn.cl/lc/cpolitica/index_html (Consultado el 10/05/2011) 
OIT (1990) Convenio 169 sobre Pubelos indigenas y Tribales en Paises Independientes http:// www.ilo.org/indigenous/Conventions/ no169/lang- - es/index.htm

(Consultado el 11/05/2011)

Peru.com (05 de junio del 2009) Presidente Alan García advierte a nativos ya está bueno de protestas" http:// www.peru.com/ noticias/portada20090605/37781/ Presidente- Alan- Garciaadvierte- a- nativos- Ya- esta- bueno- de- protestas (Consultado el 17/05/2011)

Presidencia Colombia. (1991) Constitución Política de Colombia http:// web.presidencia.gov.co/ constitucion/index.pdf (Consultado el 10/05/2011)

Tribunal Constitucional (1994) Constitución política del Perú http:// www.tc.gob.pe/ legconperu/ constitucion.html (Consultado el 10/05/2011)

\section{Sobre las autoras}

Minda Bustamante Soldevilla posee el título de abogada por la Universidad Católica Santa María de Arequipa, Perú. Karen Rosenfeld Ytier obtuvo su grado de Socióloga en la Universidad de Chile. Ambas autoras son actualmente alumnas del Magíster en Análisis Sistémico Aplicado a la Sociedad (MaSS), de la Universidad de Chile mass.facso@uchile.cl

Contacto

Magíster en Análisis Sistémico Aplicado a la Sociedad (MaSS)

Facultad de Ciencias Sociales, Universidad de Chile

Capitán Ignacio Carrera Pinto 1045

Ñuñoa, Santiago de Chile

Recibido: Julio/2011

Aceptado: Agosot/2011

Artículo disponible en: http:// www.facso.uchile.cl/publicaciones/mad/25/ bustamante05.pdf 